\title{
Language as a Mediating Factor in Interethnic Relations and Out-Group Acceptance: The Specific Case of the Malinke (Dyula) and Senoufo in Côte d'Ivoire
}

\author{
Dongui Zana Y. Ouattara \\ Alassane Ouattara University, Bouake, Côte d'Ivoire \\ Email: ouattdong@gmail.com
}

How to cite this paper: Ouattara, D.Z.Y. (2022) Language as a Mediating Factor in Interethnic Relations and Out-Group Acceptance: The Specific Case of the Malinke (Dyula) and Senoufo in Côte d'Ivoire. Open Access Library Journal, 9: e8315.

https://doi.org/10.4236/oalib.1108315

Received: December 21, 2021

Accepted: January 14, 2022

Published: January 17, 2022

Copyright ( $) 2022$ by author(s) and Open Access Library Inc.

This work is licensed under the Creative Commons Attribution International License (CC BY 4.0).

http://creativecommons.org/licenses/by/4.0/

\begin{abstract}
There is mutual acceptance between the Senoufo and Malinké, two ethnolinguistic groups of Côte d'Ivoire. In order to assess language as the mediating factor between the two groups, two different questionnaires have been submitted respectively to some individuals of each group. The statistical analysis and interpretation of the data have permitted to reveal that the Dyula language plays a key role in the peaceful relations between the Senoufo and Malinke of Côte d'Ivoire. It has been discovered that on the one hand, the bilingualism of the Senoufo draws them closer to the Malinke-Dyula. The Malinke-Dyula on the other hand develops positive attitudes and a like for the Senoufo out-group because the latter can use their preferred language.
\end{abstract}

\section{Subject Areas}

Sociolinguistics, Intercultural Communication

\section{Keywords}

Senoufo, Malinké, Multiligualism, Ethnolinguistic Group, Mutual Acceptance

\section{Introduction}

The Malinke commonly called Dyula and the Senoufo are two ethnic different ethnic groups of Côte d'Ivoire. The Senoufo ethnolinguistic which belongs to the Gur ethnic family is located in northern Côte d'Ivoire. They are renowned for their social organization which articulates around the Poro and they speak Sena- 
ri. On the other hand, the Malinke also known as Dioula and northern Mande are essentially located in northern Côte d'Ivoire as well. Their language is commonly referred to as Dyula. As opposed to the Senoufo who are originally farmers, the Malinke are mostly considered as business people and Islam is their iconic religion. According to [1], "tribal instinct" is a basic human condition. Powered by this psychological force, people are naturally prone to define themselves and others into separate categories. This categorization may result in a "them" and "us" dichotomy where "them" becomes easy to demonize; and from this, conflicts may erupt.

Indeed, between two different ethnolinguistic groups or cultural groups, problems of negative out-group attitudes may spring. However, though Senoufo and Malinke are two fundamentally different ethnolinguistic groups, there is fluid interethnic communication and proximity between them. This striking proximity between these two different groups causes the researcher to seek to understand the underlying reasons. In an attempt to probe the underlying reasons for this proximity, [2] mentions the Mandingo influence on the Senoufo through Islam. For him, Islam has played an important role in bringing the two ethnolinguistic groups closer. As an illustration of this point, people from the Ivorian southern ethnolinguistic groups often mistakenly consider the Malinke and Senoufo as the same on the basis of Islam. A closer inspection of the Senoufo ethnic group will reveal that though Islam is popularly practiced in the group, it cannot be said to be characteristic of this ethnolinguistic group. Not denying the fact that Islam may play a role in the proximity between the two ethnolinguistic groups, another factor, namely the linguistic factor needs to be assessed.

This article aims at assessing language as the mediating factor for the outgroup acceptance and relatively peaceful relationships between the two ethnolinguistic groups. This study will answer two questions: is the Dyula language a mediating factor or cement for the cohesion and mutual acceptance between the Senoufo and Malinké-Dyula? To what extent does the Dyula language reinforce the proximity between the two ethnolinguistic groups? To answer these questions, one can hypothesize that the Dyula language is a key underlying reason for the cooperation and mutual acceptance between the Senoufo and MalinkéDyula; it makes interethnic communication easier and develops positive attitudes between the two groups that result in mutual acceptance and peaceful relations. In order to check these hypotheses, a quantitative method is adopted. Two different questionnaires have been submitted respectively to some individuals of each group to collect their feedback. The study is informed by Multilingualism and Cognitive Flexibility theory and the theoretical findings of [3].

\section{Theoretical Framework}

The proximity, mutual acceptance and peaceful relations between the Senoufo and Malinke ethnolinguistic groups of Côte d'Ivoire will be explained in the light of Multilingualism and Cognitive Flexibility and the theoretical findings 
of [3]. It is worth noting that Senoufo people are supposed (or at least suspected) to understand and speak Dyula; which allows them to conduct successful interethnic communication. In other words, the Senoufo can be considered as bilinguals as they can use both the Senoufo and Dyula languages. This bilingualism of the Senoufo has implications as regards the proximity between the two ethnolinguistic groups. On the other hand, the theoretical findings of [3] support that using other people's preferred language helps in building deeper connection and out-group acceptance with them. Language and culture are connected; and because of this connection, people have a preferred language with which they have an emotional connection. This language is usually people's first language. In the context of this work, the preferred language is the one people's culture and world perception is attached to; it is the language that determines ethnic belonging. As [4] puts it, using a person's preferred language as opposed to any other will facilitate a deeper connection with that person, remove cross-cultural barriers that may separate them and thus bring them closer into mutual acceptance.

\subsection{Aron et al. (1997) Theoretical Findings}

Research performed by a group of psychologists, professors of psychology at various universities, [3] has shown that sustained, escalating, reciprocal, personalistic self-disclosure is a key factor in developing intimacy between people. People build deeper relationships when they can share that which is most important to them with other people and be understood and accepted by them [3] while close relationships also require spending time together and sharing interests, feelings, and activities, being able to communicate them is essential. In everyday life, however, disclosing important things about oneself, sharing important things about oneself in a language that is not the first or preferred language of your co-speaker may be uncomfortable or less meaningful.

A person's preferred language is the language the person is more comfortable speaking. It is the language he most dreams and thinks in. It also refers to the language he primarily heard when learning to talk. Preferred language is often interspersed with first language, with mother tongue or first language. It is worth noting here that the preferred language of an individual will not always be their first language, for example in cases where a child's family moves to another country or area soon and speaks a different language soon after they learned to speak their first language. However, it should be taken for granted that the majority of people will prefer their first language to any other. Why would a person prefer a language over another? The preference for a language stems from the fact that languages carry and transmit much more than mere words [5]. Languages go beyond transmitted mere words and structures to imply emotional connotations; cultural importance; and spiritual significance.

Then, in order to develop good relationship or intimacy with people of other cultural groups, it is important to speak and understand their preferred lan- 
guage. It is beneficial to speak and understand other people's preferred languages as this develops affective connection. This is supported by [6]; Yarrow Dunham, assistant professor of psychology and cognitive science at Yale University; and Mahzarin R. Banaji, psychology and social ethics professor at Harward University for whom this pattern is shown even in infants as they prefer people who speak languages they have heard as well as accents they have heard. A group of experts in psychology, [7] comparing monolingual and bilingual infants at six and eight months concluded that infants can distinguish between languages even as babies and then be able to tell the language they are familiar with from another one.

\subsection{Multilingualism and Cognitive Flexibility}

The first step in explaining the reason why more multilingual individuals develop more acceptance towards ethnic out-groups is their higher Cognitive Flexibility. According to [8], Cognitive Flexibility is "the awareness that in any given situation, there are options and alternatives available, the willingness to be flexible and adapt to the situation and one's self-efficacy in being flexible". This cognitive ability is increased in bilingual individuals [9], due to the flexibility requirements of language production inherent in any language [10]. Some empirical evidence supports the thesis that cognitive flexibility is influenced by language skills. It is potentially more developed when one can communicate in mutliple languages [11]. This link between multilingualism and cognitive flexibility is further supported by research comparing bilinguals and monolinguals. Examples include more effective integration of divergent data sources [12]. Considering this evidence, and since speaking multiple languages implies a greater ability to understand and represent informationin different ways, cognitive flexibility can be expected to be higher in those individuals who show a greater degree of multilingualism (Hypothesis 1). In the framework of this study, cognitive flexibility will be expected to be higher in the Senoufo as they manage to speak and understand Dyula be it perfectly or not.

Cognitive Flexibility, Out-Group Acceptance, and Deprovincialization

[13] Classic experimental research found that those who were cognitively flexible were also less prejudiced against ethnolinguistic out-group and consequently have a positive attitude towards ethnic out-groups. In a similar vein, [14] concluded that people who experience social and cultural diversity develop an increase in out-group tolerance. This is said to occur among those with the capability and motivation to integrate various kinds of conflicting information and generate new insight from earlier information, as would be implied by cognitive flexibility. While these findings are promising in explaining a link between multilingualism and out-group acceptance, further understanding of why cognitive flexibility may cause this increase in out-group acceptance is warranted.

Deprovincialization provides a potential explanation for this. Deprovincialization implies the recognition of insight into alternative cultural worldviews and a 
refraining of those held by one's in-group [15]. This concept is typically used in the context of inter-group contact (e.g. [16]), but can also be related to multilingualism through cognitive flexibility. It may be that cognitive flexibility, as both a motivated choice and ability to view information in multiple, alternative ways would make one more likely to look at life from different perspectives. Values and norms may then come to be considered somewhat arbitrary and many sets of these are viewed as equally valid. Thus, it is argued that these alternative understandings offer a basis for the insight that other groups may also perceive the world in different ways, and would further allow for reevaluation of these cultural understandings. If one does indeed also come to hold a different world view personally, this novel perception of the world would further motivate one to become more accepting of worldviews other than those held by one's ethnocultural group, since maintenance of a negative evaluation of novel worldviews might cause cognitive dissonance [17]. For these reasons, it is expected that more cognitively flexible individuals will also be more deprovincialized (Hypothesis 2).

Since deprovincialization implies relativizing one's culture, it can also entail recognition of out-group cultures as being valuable, which makes an out-group more likable [15]. Similarly, the reduction of provincialized in-group centrism may encourage one to be less restrictive with regards to which out-group one perceives oneself to share similarities with. These theoretical links are repeatedly borne out by empirical findings. [18] found evidence for deprovincialization's association with reduced out-group prejudice. Similarly, in-group reappraisal was shown to be related to lower ethnocentrism [16], and deprovincialization was found to be associated with a greater perception of a common identity [19]. Based on these theoretical arguments and empirical findings, deprovincialization is expected to be with greater out-group acceptance (Hypothesis 3). This represents a more detailed explanation of the link between cognitive flexibility and out-group acceptance as suggested by previous authors [13] [14].

All in all, it is expected that the more languages people speak, the more they will be accepting other ethnolinguistic out-groups. This is due to two sequential mechanisms: an increase in cognitive flexibility, and as a result of this, becoming more deprovincialized. Deprovincialization could in turn make a reevaluation of the out-group easier.

\section{Literature Review}

Multilingualism is the use of more than one language for communication. It is the ability of an individual to use at least one language to communicate. It has both an individual and a societal dimensions; that is why [20] defines multilingualism as "the ability of societies, institutions, groups and individuals to engage, on a regular basis, with more than one language in their day-to-day lives". Societal multilingualism is the use of more than one language in a given society. In this study, multilingualism encompasses these two dimensions. For [21], multilingualism is an occurrence regarding an individual speaker who uses at least 
two languages, a speech community where two or more languages are used. In their definition, they add that multilingualism can also be defined on two bases: the maximal competence and the minimal competence. The maximal definition means speakers are as competent and proficient in one language as they are in others. To be more specific, maximal competence stands for equal competence in all the languages. As regards the minimal definition, on the other hand, it is based on language use. It simply means the speaker is successsful enough to achieve effective communication goals. [22] conclude that multilingual speakers mostly lie between the continuum of maximal and minimal definition. However, it is worth noting that in this study, both the maximal and the minimal competences apply to the Senoufo as they can use Dyula language to communicate with the Malinke people, be it perfectly or not because, in general, most bilinguals master one language better than the other.

In multilingual settings, intergroup encounters are ubiquitous. Many cultural groups frequently experience intergroup encounters. These encounters call for more acceptance of cultural and linguistic differences in day-to-day life. Multilingualism, the ability to use at least two languages has been investigated as a factor which makes intergroup contacts easier by reducing prejudice toward out-groups and improving interethnic attitudes. Many empirical studies have shown that multilingualism is related to reducing prejuduce between different ethnolinguistic groups. To kick off, [23] addresses the significance of conciliatory language policies in defusing ethnic tensions between indigenous Kazakhs and ethnic Russians in Kazakhstan. Conciliatory language such as mild policy on ethnic ties; the unified language policy were identified as crucial to tolerant interethnic relations. By the same token, [24] convincingly argues that language and prejudice are closely related. They found out that being able to use the language of a different group reduces prejudice toward that group. Besides, [4] in his Senior Thesis (submitted in partial fulfillment of the requirement for graduation in the Honors Program) on the importance of language in cross-cultural interaction shows how being able to use the language of an out-group makes intergroup connections easier. He argues that using a person's preferred language as opposed to any other language he understands will facilitate deeper connection with that person and reduce cross-cultural barriers that may separate them.

Furthermore, [25] working on multilingualism as a predictor of acceptance of ethnic out-group argue that people who speak more languages are more cognitively flexible; that is they have an enhanced flexibility in understanding and representing information. Higher flexibility in turn is expected to be related to higher deprovincialization which results in more openness and reduced out-group dislike. More recently, [26] explores the relationship between the ability to talk to others and ethnic prejudice considering the quantity and quality of intergroup contact. A structural equal model analysis was carried out on a sample of 631 Italian citizens. The findings reveal that multilingualism leads to an increase of acceptance of intergroup differences and positive attitudes toward 
Morrocans and that the quality (but not quantity) of intergroup contact mediated those relationships. The mediating role of the quality of intergroup contact extends previous results on the relationship between multilingualism and positive attitudes toward ethnic out-group. As [27] proposes, the linguistic component is crucial in promoting co-operative diversity in multilingual settings. Being able to speak other people's language is crucial to promoting peaceful cooperation. This article explores the ability of the Senoufo to understand and use the preferred language of the Malinke-Dyula as a facilitator of the cohesion or mutual acceptance between the two groups.

\section{Method}

To conduct this study, the researcher resorts to a quantitative method. Two different questionnaires A and B are designed respectively for the Senoufo and the Malinke-Dyula. To be more specific, Questionnaire A (Appendix) was submitted to the Senoufo and Questionnaire B (Appendix) was submitted to the Malinke. In total ninety (90) participants aged at least 25 from both ethnolinguistic groups were reckoned for the investigation. Forty-five (45) Senoufo and fortyfive (45) Malinke participated in the survey. The investigation was conducted in three different places, namely Bouake, Korhogo, and Nielle, the researcher's native town, because the Senoufo and Malinke co-exist in those places. On the one hand, Nielle and Korhogo are home to the Senoufo, but also cities that host important Malinke communities as well. Bouake on the other hand is a melting-pot where you can also find important Senoufo and Malinke-Dyula communities. The participants were randomly chosen on the basis of their ethnic belonging and age; that is being Senoufo or Malinke-Dyula and aged at least 25 were the main criteria. The reason for the age criterion is that individuals aged less than 25 may not provide relevant feedback on the reasons for the proximity between the two ethnolinguistic groups. The researcher did not mind whether the participants were educated or not. In the process of data collection, the educated informants were simply given the questionnaire to read and tick the answers. As regards the uneducated, the researcher used the same questions of the questionnaire to interview them. Since they cannot read or write, the questions were translated either in Malinke-Dyula or Senoufo so as to get relevant feedback from them. The survey produced the data below. These numerical data will be analyzed and interpreted.

\subsection{The Senoufo "Bilingualism" in Interethnic Relations}

Table 1. Participants' ability to speak Dyula.

\begin{tabular}{ccc}
\hline Answers & Number of informants & $\%$ \\
\hline Yes & 43 & $95.66 \%$ \\
No & 2 & $4.44 \%$ \\
TOTAL & 45 & $100 \%$ \\
\hline
\end{tabular}


Table 2. Senoufo-Malinke Cohesion.

\begin{tabular}{ccc}
\hline Answers & Number of informants & $\%$ \\
\hline Yes & 45 & $100 \%$ \\
No & 0 & $0 \%$ \\
TOTAL & 45 & $100 \%$ \\
\hline
\end{tabular}

Table 3. Factors of the Senoufo-Malinke cohesion

\begin{tabular}{ccc}
\hline Factors & Number of informants & $\%$ \\
\hline Last names & 5 & $11.11 \%$ \\
language & 33 & $73.33 \%$ \\
Religion & 7 & $15.56 \%$ \\
Clothing & 0 & $0 \%$ \\
TOTAL & 45 & $100 \%$ \\
\hline
\end{tabular}

\subsection{Linguistic Factor in the Malinke-Senoufo Relations}

Table 4. The language of interethnic communication.

\begin{tabular}{ccc}
\hline Languages & Number of informants & $\%$ \\
\hline Senoufo & 2 & $4.45 \%$ \\
Malinke-Dyula & 37 & $82.22 \%$ \\
French & 6 & $13.36 \%$ \\
TOTAL & 45 & $100 \%$ \\
\hline
\end{tabular}

Table 5. How the Malinke feel about the Senoufo.

\begin{tabular}{ccc}
\hline Feelings & Number of informants & $\%$ \\
\hline Feel indifferent & 0 & $0 \%$ \\
Feel happy & 16 & $35.56 \%$ \\
Feel closer to & 29 & $64.44 \%$ \\
TOTAL & 45 & $100 \%$ \\
\hline
\end{tabular}

Table 6. How the Malinke perceive the Senoufo.

\begin{tabular}{ccc}
\hline Perceive the Senoufo as: & Number of informants & $\%$ \\
\hline people of other ethnic groups & 0 & $0 \%$ \\
brothers and sisters & 41 & $91.11 \%$ \\
friends & 4 & $8.89 \%$ \\
TOTAL & 45 & $100 \%$ \\
\hline
\end{tabular}

\section{Analysis and Interpretation}

\subsection{The Senoufo "Bilingualism" in the Malinke-Senoufo Relations}

The first thing that is worth mentioning here is the ability of the Senoufo to un- 
derstand and use Malinke-Dyula in addition to their own language of identification. As can be seen in Table 1, only two (2) of the forty-five (25) Senoufo people investigated declare they cannot speak Malinke-Dyula. On the other hand, $95.56 \%$ of them asserted their ability to understand and speak Malinke-Dyula. This is illustrative of the ability of the Senoufo to communicate in Dyula language in general. Indeed, one can say that at least every Senoufo individual is supposed to understand and speak Malinke-Dyula be it perfectly or not. This is so much true that in casual contexts, the Malinke people tend to address the Senoufo individuals in Malinke-Dyula. Likewise, the Senoufo people will address the Malinke-Dyula (especially the uneducated) in the latter's language. This tendency is general heterogenous casual contexts that gather individuals of both ethnolinguistic groups. It is true that people from other ethnolinguistic groups may understand and speak Malinke-Dyula; but not in equal proportion as the Senoufo. The Senoufo people have a particular attitude to this language; it is the reason why people from other ivorian ethnic groups often consider Malinke-Dyula as the speech of the Senoufo. The reason for this multilingualism of the Senoufo may include early exposure to the Malinke-Dyula language in some geographical areas; the geographical proximity between the two ethnolinguistic groups; and possible economic dividends that the Senoufo may benefit from this language.

No matter the reasons for this "bilingualism" of the Senoufo, the relations between the Senoufo and the Malinke-Dyula are relatively peaceful. There is an apparent closeness; cohesion or mutual acceptance between the two ethnolinguistic groups. This is recognized by $100 \%$ of the Senoufo informants as shown in Table 2. One may wonder what factor accounts for this proximity between these two fundamentally and culturally different ethnolinguistic groups. A set of four (4) factors, namely last names, language and religion (Islam) and clothing style have been proposed for the Senoufo informants to choose from. Let us kick off with last names. Many people consider the Senoufo and Malinke-Dyula as the same group because they share some last (family names). This is believed by five (5) out of forty-five Senoufo informants; that is $11.11 \%$. This belief is often shared by the people of other ethnolinguistic groups of Côte d'Ivoire for whom the Senoufo ans Malinke are the same. Indeed, two individuals respectively Senoufo and Malinke may share Kone as their last name and claim to belong to culturally different ethnolinguistic groups. This may be due to some historical circumstances and a said "colonization" of the Senoufo by the Malinke-Dyula. Likewise, religion is often viewed by many as a factor that brings these ethnolinguistic groups closer. Seven (7) or $15.56 \%$ as Table 3 reads side with this belief. Indeed, a great number of Senoufo people practice Islam as their religion; and this combined with the last names and given names often borne by the Senoufo drive many Ivorian people from other ethnolinguistic groups to think that Islam is par excellence the religion of identification of the Senoufo, which is far from truth. It can be noticed that a large number of Senoufo individuals who 
bear Malinke-Dyula last names and muslim names practice other religions, which shows that the religious label stuck on the Senoufo as muslims on the basis of some names is wrong. Moreover, clothing line is often reckoned by some individuals as a factor which accounts for the proximity between the Senoufo and Malinke-Dyula. This is not relevant as it is more apparent than real. It is rather a manifestation of the proximity between the two cultural groups; that is why, none of the Senoufo participants has ticked it as an underlying factor for the proximity between the two ethnolinguistic groups.

As can be seen, neither of last names; religion (Islam) or clothing line are considered by the majority of the informants as factors that make the cement between the two groups. Contrarily, language factor has been pointed to by the large majority of the informants. As a matter of fact, thirty three (33) participants, which corresponds to $73.33 \%$ have agreed that Malinke-Dyula language is the bridge between the two communities. This can better be understood in the light of multilingualism and cognitive flexibilty theory. As a reminder, this theory posits that multilingual individuals develop more acceptance towards ethnic out-group through their higher cognitive flexibility. As [9] put it, cognitive ability or flexibility is increased in bilinguals. It means that since the Senoufo are bilinguals (multilinguals), their cognitive flexibility is higher, especially towards the Malinke-Dyula. In line with [10], cognitive flexibility is developed in the Senoufo because they can communicate in two languages, Senoufo and Malinke-Dyula languages. The Senoufo are cognitively flexible and consequently less prejudiced against the Malinke-Dyula ethnolinguistic out-group, which justifies their positive and like for the Malinke-Dyula ethnolinguistic out-group. This can be bolstered by [14] who conclude that people who experience social and cultural diversity through multilingualism develop an increase in out-group tolerance. In other words, the bilingualism of the Senoufo is the basis of their deprovincialization.

As a reminder, deprovincialization implies revitalizing one's culture and the recognition of the out-groups' cultures as being valuable which makes an outgroup more likable [15]. The ability of the Senoufo to speak Malinke-Dyula language in addition to their own ethnic language of identification drives them to relativize their culture and thus recognize the Malinke out-group as valuable and more likable. The reduced Senoufo provincialized or prejudiced in-group centrism also accounts for the closeness between the two ethnolinguistic groups. The centrality of the linguistic factor in the intergroup relationship between the two groups is such that even religion that some people consider as the cement or glue between them is in fact grounded on language. Indeed, it is worth noting that in intergroup religious activities or ceremonies, the language which is usually used is Dyula as the Malinke people take it for granted that the Senoufo people can understand and speak their language. However, a change is being operated at this level as more and more Senoufo religious guides have started preaching in their own language especially in social settings essentially manned 
by Senoufo individuals, not forgetting of course to translate into Malinke-Dyula for others to understand. It is as if Malinke-Dyula language has become or is becoming the second language of the Senoufo.

\subsection{The Linguistic Factor in the Malinke-Senoufo Relations}

In general, as opposed to the Senoufo, the Malinke-Dyula do not speak the Senoufo language. It is true that some of them may understand and speak Senoufo; but this is not spread community-wide as the Malinke-Dyula language in the Senoufo ethnolinguistic group. It seems that since the Senoufo are able to understand and speak their language, they feel it is no use to learn the latter's language. However, they are very close to the Senoufo. This is illustrated by one female Malinke participant who said "when I see a Senoufo, I see one of our kind". There are several factors for this. In Questionnaire B (see Appendix), the Malinke-Dyula informants were asked which language the Senoufo use to communicate with them during interethnic encounters. For this, three (3) languages namely, French; Senoufo; and Dyula were submitted. As can be seen in Table 4, the Senoufo language is not used as the language of interethnic communication in casual encounters. This is illustrated by the fact that only two (2) informants consider that they use Senoufo in interactions. These two (2) participants are no doubt individuals who understand and use the Senoufo language. In proportion, they represent $4.45 \%$ which is not enough to consider Senoufo as the language of interethnic communication. The Senoufo language is rarely heard during casual encounters between the individuals of the two groups. As to French language, some six (6) participants, corresponding to $13.36 \%$ of the total number (45) say it is their language of communication during interethnic encounters between the individuals of the two groups. As a matter of fact, the educated Senoufo and Malinke-Dyula individuals most often prefer using French in their interactions.

However, the language that the Senoufo frequently uses to communicate with the Malinke is the Dyula language. This can be observed during casual contexts gathering individuals of the two groups. As Table 4 reads, the large majority of the participants (82.22\%) acknowledge this. Indeed, in their large majority, the Senoufo people will use Malinke-Dyula to communicate in intergroup casual settings. This language is obviously the preferred language of the Malinke people. And using their preferred language produces emotional or affective feelings. To check this, the Malinke informants were asked how they feel about the Senoufo when the latter use their preferred language to interact with them. Three feelings (feel indifferent; feel happy; feel closer to them) were proposed. As can be seen, in Table 5, none (0\%) of the Malinke participants feel indifferent to this. Sixteen (16) or $35.56 \%$ say they feel happy and twenty-nine (29) of them (64.44\%) assert they feel closer to the Senoufo as they use their preferred language of identification and communication. This reveals how important speaking to other people in their preferred language is. Late South African President and Peace Nobel Prize winner Nelson Mandela once emphasized that if you 
speak to someone in a language they understand, this goes to the head; if you speak to them in their language, this goes to their heart. This can be paraphrased or adapted saying that if you speak to someone in a language they understand, this goes to their head; but if you speak to them in their preferred language, this goes to their heart and obviously produces positive emotional feelings including the ones the Malinke-Dyula informants mention here. Those feelings cause them to have a certain perception of the Senoufo ethnolinguistic out-group.

Thus, when asked how they perceive the Senoufo, none of them perceive them as ordinary people of other ethnic groups who do not speak Dyula. One can read in Table 6 that our (4) participants (8.89\%) say they view them as friends; and the large majority $(91.11 \%)$ assert they view the Senoufo people as brothers and sisters. These figures apparently show that the use of the Dyula language by the Senoufo during intergroup encounters develops a like of the Malinke for them. In other words, the use of Dyula language for interethnic communication between the Senoufo and Malinke plays an important role the intergroup relations. In fact, using other people's preferred language is crucial because language is more than just the words used to communicate. It has emotional connotations and cultural importance. Even if people can communicate proficiently in a second or other languages, there are still benefits to speaking to them in their preferred language. This can be sustained with [3] theoretical findings. According to them, people build deeper relationships when they can share that which is important to them with other people and be understood and accepted by them. But sharing important things about oneself in a language that is not the first or preferred language of your co-speaker may be less meaningful. Then, in order to develop close relationship or intimacy with people of other cultural groups, it is important to speak and understand their preferred language. The Senoufo people can understand and use Dyula language; then, this can account for the good relationships between the two ethnolinguistic groups. Actually, it is beneficial to speak other people's preferred language; this develops affective connection. Cognitive psychologist eminent [28] sides with this posit.

\section{Key Findings}

On the one hand, the analysis and interpretation of the data has confirmed the "bilingualism" of the Senoufo who speak Senoufo and can on average understand and speak Dyula perfectly or not. There is mutual acceptance between the Senoufo and the Malinke-Dyula, and the "bilingualism" of the Senoufo is a key reason for this. The deeper explanation for this lies in the posits of multilingualism and cognitive flexibility theory which shows that the deprovincialization of the Senoufo underlied by their ability to understand and use Dyula draws them closer to the Malinke-Dyula and develop a like for the latter. This out-group like accounts for the peaceful relations between the two groups. These findings are in line with the results of researchers like [24] [25] and [26]. In fact, [24] found that being able to use the language of an out-group reduces prejudice towards that 
group. In the light of this study, it can be understood that the ability of the Senoufo to use Dyula language has reduced their prejudiced views about the Malinke-Dyula ethnolinguistic group. "Bilingualism" makes them more cognitively flexible; deprovincialized; and stands as a catalyst of mutual acceptance between the two ethnic groups [25]. Likewise, [26] in investigating the Italians and Morrocans found similar results as it was discovered that multilingualism led to an increase of acceptance and positive attitudes towards Morrocans.

On the other hand, the ability of the Senoufo to communicate with the Malinke-Dyula in their preferred language is beneficial to the relationship between the two cultural groups. This is admitted by all the Malinke-Dyula participants who confirmed that this ability of the Senoufo to use their preferred language produces positive emotional feelings in them. It makes them feel happy; closer to the Senoufo out-group; and eventually leads them to mostly view the Senoufo as brothers and sisters. Thus, these findings also strengthens [3] theoretical findings that being able to communicate using the preferred language of other cultural groups develops affective connections and positive attitudes.

\section{Conclusions}

The striking proximity or mutual acceptance between the Senoufo and the Malinke-Dyula, two fundamentally different ethnolinguistic groups of Côte d'Ivoire has caused the researcher to seek to probe the underlying reasons. Thus in a study on the language factor as a mediating factor in the interethnic relations between the Senoufo and Malinke has been carried out. It was meant to highlight or answer two main research questions, whether the linguistic component constitutes the cement between the two cultural groups and how language operates. It was hypothesized that the Dyula language is a ground for cooperation; strong cohesion; and mutual acceptance between the two ethnolinguistic groups as it makes the interethnic communication easier between them and draws them closer. The chief purpose was to assess language as the bedrock of the relatively peaceful relations between the two ethnolinguistic groups and see if the different ethnic groups of Côte d'Ivoire should be encouraged into learning one another's languages to better interethnic relations. To achieve this goal, the researcher resorted to a quantitative study in which he submitted two different questionnaires respectively to the Some Senoufo and Malinke-Dyula people to collect the data.

Statistical analysis and interpretation of these data have permitted to verify the hypotheses and answer the research questions. It has been discovered on the one hand that the "bilingualism" of the Senoufo; that is their ability to speak Dyula on top of Senoufo is an explanation of the proximity between the two ethnolinguistic groups. On the other hand, it was found that the Malinke-Dyula develop a like for the Senoufo because the latter can use their preferred language for interethnic communication. It is thus apparent that language, the Dyula language mediates as the factor that draws the two ethnolinguistic groups closer as it makes interethnic communication and fosters tolerance and mutual acceptance 
easier. In the light of this, one can side with [27] that the linguistic component is critical in promoting co-operative diversity in multilingual settings. If so, in multiethnic societies, language policies need to be adapted or reconsidered to prevent misunderstandings and conflicts that may spring from poor interethnic relations and prejudice. A country like Côte d'Ivoire should promote individual multilingualism and encourage the different ethnolinguistic groups to learn one another's languages to boost interethnic co-operation.

\section{Conflicts of Interest}

The author declares no conflicts of interest.

\section{References}

[1] Van Vugt, M. and Park, J.H. (2010) The Tribal Instinct Hypothesis: Evolution and Social Psychology of Intergroup Relations. In: Sturmer, S. and Snyder, M., Eds., The Psychology of Prosocial Behavior: Group Processes, Intergroup Relations, and Helping, Wiley-Blackwell, Hoboken, 13-32. https://doi.org/10.1002/9781444307948.ch1

[2] Coulibaly, N.F. (2010) L'Influence de la Culture Mandingue à travers le Processus d'Islamisation de la Société Senoufo de Côte d'Ivoire, des origines à nos jours. $R e$ vue Africaine d Anthropologie, Nyansa-Pô, No. 10, 61-79.

[3] Aron, A., Melinat, E., Aron, E.N., Vallone, R.D. and Bator, R.J. (1997) The Experimental Generation of Interpersonal Closeness: A Procedure and Some Preliminary Findings. Personality and Social Psychology Bulletin, 23, 363-377. https://doi.org/10.1177/0146167297234003

[4] Norton, L. (2018) The Importance of Language in Cross-Cultural Interaction. A Senior Thesis Submitted in Partial Fulfillment of the Requirements for Graduation in the Honors Program of Liberty University, Lynchburg.

[5] Davis, T.Y. and Moore, W.L. (2014) Spanish Not Spoken Here: Accounting for the Racialization of the Spanish Language in the Experiences of Mexican Migrants in the United States. Ethnicities, 14, 676-697. https://doi.org/10.1177/1468796814523740

[6] Oludamini, O., Dunham, Y. and Banaji, M.R. (2010) The Language of Implicit Preferences. Journal of Experimental Social Psychology, 46, 999-1003. https://doi.org/10.1016/j.jesp.2010.07.006

[7] Weikum, W.M., Vouloumanos, A., Navarra, J., Soto-Faraco, S., Sebastian Galles, N. and Werker, J.F. (2007) Visual Language Discrimination in Infancy. Science, 316, 1159. https://doi.org/10.1126/science.1137686

[8] Martin, M. and Anderson, C.M. (1998) The Cognitive Flexibility Scale: Three Validity Studies. Communication Reports, 11, 1-9. https://doi.org/10.1080/08934219809367680

[9] Adesope, O.O., Lavin, T., Thompson, T. and Ungerleider, C. (2010) A Systematic Review and Meta-Analysis of Cognitive Correlates of Bilingualism. Review of Educational Researches, 80, 207-245. https://doi.org/10.3102/0034654310368803

[10] Martin, M. and Rubbin, R. (1995) A New Measure of Cognitive Flexibility. Psychological Reports, 76, 623-626. https://doi.org/10.2466/pr0.1995.76.2.623

[11] Kozulin, A. (1999) Profiles of Immigrant Students' Cognitive Performance on Raven's Progressive Matrices. Perceptual and Motor Skills, 87, 1311-1314. 
https://doi.org/10.2466/pms.1998.87.3f.1311

[12] Kharkhurin, A.V. (2008) The Effect of Linguistic Proficiency, Age of Second Language Acquisition, and Length of Exposure to a New Cultural Environment on Bilinguals' Divergent Thinking. Biligualism and Cognition, 11, 225-243. https://doi.org/10.1017/S1366728908003398

[13] Rokeach, M. (1948) Generalized Mental Rigidity as a Factor in Ethnocentrism. The Journal of Abnormal and Social Psychology, 43, 259-278. https://doi.org/10.1037/h0056134

[14] Crisp, R.J. and Turner, R.N. (2011) Cognitive Adaption to the Experience of Social and Cultural Diversity. Psychological Bulletin, 137, 242-266.

https://doi.org/10.1037/a0021840

[15] Pettygrew, T.F. (1997) Generalized Intergroup Contact Effects on Prejudice. Personality and Social Psychology Bulletin, 23, 173-185. https://doi.org/10.1177/0146167297232006

[16] Verkuyten, M., Thijs, J. and Bekhuis, H. (2010) Intergroup Contact and Intergroup Reappraisal: Examining the Deprovincialization Thesis. Social Psychology Quarterly, 73, 398-416. https://doi.org/10.1177/0190272510389015

[17] Elliot, A.J. and Devine, P.G. (1994) On the Motivational Nature of Cognitive Dissonance: Dissonance as Psychological Discomfort. Journal of Personality and Social Psychology, 67, 382-394. https://doi.org/10.1037/0022-3514.67.3.382

[18] Martinovic, B. and Verkuyten, M. (2013) We Were the First, So We Determine the Rules of the Game: Autochthony and Prejudice towards Out-Groups. European Journal of Social Psychology, 43, 637-647. https://doi.org/10.1002/ejsp.1980

[19] Verkuyten, M. and Martinovic, B. (2015) Majority Member's Recognition and Protest against Discrimination of Immigrants: The Role of Power Threat, Deprovincialization and Common National Identity. Social Justice Research, 28, 257-273. https://doi.org/10.1007/s11211-015-0248-4

[20] Cenoz, J. (2013) The Influence of Bilingualism on Third Language Acquisition: Focus on Multilingualism. Language Teaching, 46, 71-86. https://doi.org/10.1017/S0261444811000218

[21] Stridhar, K. (1996) Societal Multilingualism. In: McKay, S.L. and Hornberger, N.H., Eds., Sociolinguistics and Language Teaching, Cambridge University Press, Cambridge, 47-70. https://doi.org/10.1017/CBO9780511551185.005

[22] Cook, V. and Basseti, B. (Eds.) (2011) Relating Language and Cognition: The Second Language User. In: Cook, V. and Basseti, B., Eds., Language and Bilingual Cognition, Psychology Press, New York, 149-190.

[23] Lee, C. (2004) Languages and Ethnic Politics in Central Asia: The Case of Kzakhstan. Journal of International and Area Studies, 11, 101-116.

[24] Collins, K.A. and Clement, R. (2012) Language and Prejudice Direct and Moderated Effects. Journal of Language and Social Psychology, 31, 376-396. https://doi.org/10.1177/0261927X12446611

[25] Kieran, D.M. and Martinovic, B. (2018) Multilingualism and Out-Group Acceptance: The Mediating Roles of Cognitive Flexibility and Deprovincialization. Journal of Language and Social Psychology, 37, 51-73. https://doi.org/10.1177/0261927X17706944

[26] Servidio, R., Giammusso, I., Boca, S. and Mirisola, A. (2021) Multilingualism and Ethnic Prejudice: The Mediating Roles of Intergroup Contact. Social Psychology, 52, 90-100. https://doi.org/10.1027/1864-9335/a000438 
[27] Stephen, O.O. (2013) The Language Factor in the Search for National Cohesion and Integration in Kenyab. The East African Review, 47, 57-69.

https://doi.org/10.4000/eastafrica.406

[28] Reiman, A.-K., Dunham, Y., Merrillm A., Hoosain, L. and Olson, K.R. (2010) Preference of High Status Groups. Developmental Psychology, 50, 1081-1090.

https://doi.org/10.1037/a0035054 


\section{Appendix}

\section{Questionnaire A}

This questionnaire is submitted to some Senoufo people to have their opinion about some questions related to the cohesion and mutual acceptance between them and the Malinke-Dyula communities (Read and check the answers to your convenience and justify if necessary).

I. Identification of the informants
1. Are you Senoufo?
YES
NO
2. Gender:
Male
Female
3. Age $[25-35]$
[45 - 55]
[35 - 45]
[55 - 65]

II. The Senoufo Bilingualism in the Senoufo-Malinke-Dyula relations

1. Do you speak Senoufo?

YES

NO $\square$

2. Can you speak Malinké-Dyula language?

YES

$\mathrm{NO}$

3. Do you think there is proximity and mutual acceptance between the Senoufo and the Malinke-Dyula?

YES

NO $\square$

4. Which of the factors below do you think best accounts for this cohesion and mutual acceptance?

Some last names

Malinke-Dyula Language

Religion (Islam)

Clothing line

Justify

\section{Questionnaire B}

This questionnaire is submitted to some Malinke-Dyula people to have their opinion about some questions related to the cohesion and mutual acceptance between them and the Senoufo communities (Read and check the answers to your convenience and justify if necessary).

I. Identification of the informants
4. Are you Malinke-Dyula?
YES
NO
5. Gender:
Male
Female
6. Age [25-35]
[45 - 55]
[35 - 45]
[55 - 65]

II. The Dyula language in the proximity between the Malinke-Dyula and the Senoufo

1. Do you speak Malinke-Dyula? YES

NO

2. What language do the Senoufo people frequently use to interact with you in informal context?

Senoufo

Malinke-Dyula

French 
3. How do you feel when the Senoufo interact with you in your language? You feel indifferent You feel happy

You feel closer to them

4. How do you consider the Senoufo people?

You consider them as people of other ethnic groups

You consider them as brothers and sisters

You consider them as friends 